\title{
Spontaneous polarization in the cluster ferroelectric system close to the phase transition point
}

\author{
N.A.Korynevskii \\ Institute for Condensed Matter Physics \\ of the National Academy of Sciences of Ukraine, \\ 1 Svientsitskii Str., 79011 Lviv, Ukraine
}

Received April 22, 1999

\begin{abstract}
A two-stage layer by layer integration of the ferroelectric two-particle cluster system partition function functional is made in the quartic basic distribution of the dipole momentum fluctuation approximation. The shape of the layers of integration is determined by peculiarities of fluctuation processes (of the Gaussian and the non-Gaussian types) for separate subsets of collective variables. The minimization procedure of the partition function functional unintegrated over one variable (with quasimomentum and Matsubara's frequency equal to zero) gives an equation for the order parameter (i.e., the spontaneous polarization) of the system. Solutions of this equation at various values of system parameters and external field are found in the $T_{\mathrm{c}}$ neighbourhood.
\end{abstract}

Key words: functional, cluster ferroelectrics, phase transition, polarization

PACS: $75.40 . C x$

Among various problems of investigating the real physical systems close to the phase transition point, namely calculating their universal and non-universal characteristics, the problem of the equation of state is a unique one. This is due to the fundamental role of such an equation for a complete description of thermodynamic properties of the system.

The method of collective variables applied in the Ising model theory [1] has opened new possibilities in this area. The method enables us to provide a direct calculation of characteristic functions based on the equilibrium statistical physics.

In the papers $[2,3]$ an essential approach was made to obtain a free energy of the ferroelectric cluster system in the phase transition point neighbourhood. In order to obtain an equation of state (i.e., an equation for spontaneous polarization) it is necessary to integrate over all collective variables in the partition function functional with the exception of only one variable with momentum and Matsubara's frequency equal to zero. The mean value of this variable is, in fact, the order parameter of the 
system.

We shall regard a two-particle cluster ferroelectric system in the external electric field $\vec{h}$ parallel to the $z$-axis. The short-range intracluster interactions $V$ and the transverse electric field $\Gamma$ will be taken as a reference system. Using generalized transition operators

$$
Y_{\lambda}\left(\vec{R}_{q}\right)=\sum_{m} U_{\lambda m} X^{m}\left(\vec{R}_{q}\right)
$$

where $X^{m}\left(\vec{R}_{q}\right)$ are well-known Hubbard-Stasyuk operators [4,5], for the quartic form of the partition function functional in the collective variables representation we obtain:

$$
\begin{aligned}
& Z=Z_{0}^{N} \int\left(\mathrm{d} \rho_{\lambda}(\vec{k}, \nu)\right)^{N} \exp \left\{\sum _ { \lambda = 1 , 5 } \sum _ { k , \nu } \left[\frac{\beta}{2} \Phi_{\lambda}(\vec{k}) \rho_{\lambda}(\vec{k}, \nu) \rho_{\lambda}(-\vec{k},-\nu)-\right.\right. \\
& \left.\left.-2 \beta \sqrt{N} h \rho_{\lambda}(\vec{k}, \nu) \delta_{\lambda 1} \delta(\vec{k}) \delta(\nu)\right]\right\} \\
& \times \int\left(\mathrm{d} \omega_{\lambda}(\vec{k}, \nu)\right)^{N} \exp \left\{-\frac{(2 \pi)^{2}}{2} \sum_{\lambda=1,5} \sum_{k, \nu} \mathcal{M}_{\lambda \lambda}(\vec{k}, \nu,-\vec{k},-\nu) \omega_{\lambda}(\vec{k}, \nu) \omega_{\lambda}(-\vec{k},-\nu)\right. \\
& \left.+\frac{(2 \pi)^{4}}{4 !} \sum_{\lambda=1,5} \sum_{\substack{k_{1}, \ldots, k_{4} \\
\nu_{1}, \ldots, \nu_{4}}} \mathcal{M}_{\lambda \lambda \lambda \lambda}\left(\vec{k}_{1}, \nu_{1}, \ldots \vec{k}_{4}, \nu_{4}\right) \omega_{\lambda}\left(\vec{k}_{1}, \nu_{1}\right) \ldots \omega_{\lambda}\left(\vec{k}_{4}, \nu_{4}\right)\right\} .
\end{aligned}
$$

Here

$$
Z_{0}=\sum_{i=1}^{4} \exp \left(-\beta E_{i}\right)=2\left(\cosh \beta V+\cosh \beta \sqrt{V^{2}+4 \Gamma^{2}}\right)
$$

is the non-interacting clusters partition function;

$$
E_{1}=\sqrt{V^{2}+4 \Gamma^{2}}, \quad E_{2}=-\sqrt{V^{2}+4 \Gamma^{2}}, \quad E_{3}=-V, \quad E_{4}=V
$$

is the reference system energy spectrum; $\rho_{\lambda}(\vec{k}, \nu)$ are the collective variables (corresponding to generalized transition operators $Y_{\lambda}\left(\vec{R}_{q}\right)(1)$ in a frequency-momentum representation); $\omega_{\lambda}(\vec{k}, \nu)$ are the variables conjugated to $\rho_{\lambda}(\vec{k}, \nu) ; \Phi_{\lambda}(\vec{k})$ are the Fourier transforms of the $\lambda$-th eigenvalue of the intercluster dipole-dipole potential. Summation in (2) over $\vec{k}$ must be done inside the first Brillouin zone and over $\nu$ from zero to infinity. We would like to note that expression (2) differs from the one presented in [2] by the presence of the term proportional to an external field $\vec{h}$. In [6] such a field was taken into the reference system, but here we shall regard it in the framework of the collective variables method.

With the accuracy up to the fourth order inclusive the non-equal to zero coefficients (i.e., cluster cumulants) $\mathcal{M}_{\lambda \ldots \lambda}\left(\vec{k}_{1}, \nu_{1} \ldots \vec{k}_{n}, \nu_{n}\right)$ are:

$$
\begin{aligned}
& \mathcal{M}_{\lambda \lambda}\left(\vec{k}_{1}, \nu_{1}, \vec{k}_{2}, \nu_{2}\right)=\frac{E_{1}^{2}-E_{3}^{2}}{2 \beta E_{1} Z_{0}}\left[\frac{\mathrm{e}^{\mp \beta E_{3}}-\mathrm{e}^{-\beta E_{1}}}{\left(E_{1} \mp E_{3}\right)^{2}+\nu^{2}}+\frac{\mathrm{e}^{-\beta E_{2}}-\mathrm{e}^{\mp \beta E_{3}}}{\left(E_{2} \mp E_{3}\right)^{2}+\nu^{2}}\right] \\
& \times \delta\left(\vec{k}_{1}+\vec{k}_{2}\right) \delta\left(\nu_{1}+\nu_{2}\right),
\end{aligned}
$$




$$
\begin{aligned}
& \mathcal{M}_{\lambda \lambda \lambda \lambda}\left(\vec{k}_{1}, \nu_{1}, \ldots, \vec{k}_{4}, \nu_{4}\right)=\frac{E_{1}^{2}-E_{3}^{2}}{4 \beta^{3} E_{1}^{2} Z_{0}}\left[\frac{\mathrm{e}^{\mp \beta E_{3}}-\mathrm{e}^{-\beta E_{1}}}{\left(E_{1} \mp E_{3}\right)^{2}+\nu^{2}}+\frac{\mathrm{e}^{-\beta E_{2}}-\mathrm{e}^{\mp \beta E_{3}}}{\left(E_{2} \mp E_{3}\right)^{2}+\nu^{2}}\right] \\
& \times\left[\frac{\left(E_{1} \mp E_{3}\right)\left(\cosh \beta\left(E_{2} \mp E_{3}\right)-1\right)}{\left(E_{2} \mp E_{3}\right)^{2}+\nu^{2}}-\frac{\left(E_{1} \mp E_{3}\right)\left(\cosh \beta\left(E_{1} \mp E_{3}\right)-1\right)}{\left(E_{1} \mp E_{3}\right)^{2}+\nu^{2}}\right] \\
& \times \delta\left(\vec{k}_{1}+\ldots+\vec{k}_{4}\right) \delta\left(\nu_{1}+\ldots+\nu_{4}\right)-3 \mathcal{M}_{\lambda \lambda}^{2} ;
\end{aligned}
$$

the upper sign in (5) corresponds to $\lambda=1$ and the lower one corresponds to $\lambda=5$, respectively, and for simplification we put $\nu_{i}^{2}=\nu^{2}$. It must be emphasized that complete Matsubara's frequency dependent expressions (5) for a two-particle cluster ferroelectric system are found here for the first.

When $\Gamma=0$,

$$
\begin{aligned}
& \mathcal{M}_{\lambda \lambda}\left(\vec{k}_{1}, \nu_{1}, \vec{k}_{2}, \nu_{2}\right)= \begin{cases}\frac{\exp ( \pm \beta V)}{4 \cosh \beta V} \delta\left(\vec{k}_{1}+\vec{k}_{2}\right) \delta\left(\nu_{1}+\nu_{2}\right), & \nu=0, \\
0, & \nu \neq 0 ;\end{cases} \\
& \mathcal{M}_{\lambda \lambda \lambda \lambda}\left(\vec{k}_{1}, \nu_{1}, \ldots, \vec{k}_{4}, \nu_{4}\right)= \begin{cases}\frac{1-2 \mathrm{e}( \pm 2 \beta V)}{16 \cosh ^{2} \beta V} \delta\left(\vec{k}_{1}+\ldots+\vec{k}_{4}\right) \delta\left(\nu_{1}+\ldots+\nu_{4}\right), & \nu=0 \\
0, & \nu \neq 0 .\end{cases}
\end{aligned}
$$

When $V=0$,

$$
\begin{aligned}
\mathcal{M}_{\lambda \lambda}\left(\vec{k}_{1}, \nu_{1}, \vec{k}_{2}, \nu_{2}\right)= & \frac{\Gamma \sinh 2 \beta \Gamma}{\beta\left(4 \Gamma^{2}+\nu^{2}\right)(\cosh 2 \beta \Gamma+1)} \delta\left(\vec{k}_{1}+\vec{k}_{2}\right) \delta\left(\nu_{1}+\nu_{2}\right), \\
\mathcal{M}_{\lambda \lambda \lambda \lambda}\left(\vec{k}_{1}, \nu_{1}, \ldots, \vec{k}_{4}, \nu_{4}\right)= & \frac{\Gamma \sinh ^{2} 2 \beta \Gamma(\sinh 2 \beta \Gamma-3 \beta \Gamma)}{\beta^{3}\left(4 \Gamma^{2}+\nu^{2}\right)(\cosh 2 \beta \Gamma+1)^{2}} \\
& \times \delta\left(\vec{k}_{1}+\ldots+\vec{k}_{4}\right) \delta\left(\nu_{1}+\ldots+\nu_{4}\right)
\end{aligned}
$$

both for $\lambda=1$ and $\lambda=5$.

Among two branches of the collective vibrations in (2) there is only one $(\lambda=1)$ responsible for the ferroelectric phase transition [2]. So, $\left\langle Y_{1}\left(\vec{R}_{q}\right)\right\rangle$ is really an order parameter of the ferroelectric cluster system.

As far as variables $\rho_{5}(\vec{k}, \nu)$ are non-active in the phase transition process, the curve of their distribution is insensitive to the temperature, remaining the Gaussian one. So, integration $(2)$ over $\rho_{5}(\vec{k}, \nu)$ must be done using Gaussian measure density:

$$
f^{G}\left(\omega_{5}\right)=\exp \left\{-\frac{(2 \pi)^{2}}{2} \mathcal{M}_{55}(\vec{k}, \nu,-\vec{k},-\nu) \omega_{5}(\vec{k}, \nu) \omega_{5}(-\vec{k},-\nu)\right\} .
$$

Quite different measure density is adequate with reference to $\rho_{1}(\vec{k}, \nu)$ variables. Due to their role in the ferroelectric phase transition, those variables are distributed according to non-Gaussian law, and change the form of their distribution when temperature passes $T_{\mathrm{c}}$. We shall use quartic measure density, the simplest one which correctly describes the order parameter fluctuations near phase transition point:

$$
\begin{array}{r}
f^{q}\left(\omega_{1}\right)=\exp \left\{-\frac{(2 \pi)^{2}}{2} \mathcal{M}_{11}(\vec{k}, \nu,-\vec{k},-\nu) \omega_{1}(\vec{k}, \nu) \omega_{1}(-\vec{k},-\nu)\right) \\
\left.+\frac{(2 \pi)^{4}}{4 !} \mathcal{M}_{1111}\left(\vec{k}_{1}, \nu_{1}, \ldots, \vec{k}_{4}, \nu_{4}\right) \omega_{1}\left(\vec{k}_{1}, \nu_{1}\right) \ldots \omega_{1}\left(\vec{k}_{4}, \nu_{4}\right)\right\} .
\end{array}
$$


Because ferroelectric order parameter is determined at zero Matsubara's frequency, it was shown [2] that measure density (9) may be taken at $\nu_{i}=0$. The discrete spectrum of $\nu$ and gap between $\nu=0$ and $\nu=\frac{2 \pi}{\beta} n$ at any $n= \pm 1, \pm 2, \ldots$, confirm this statement.

It is well known that integration with the Gaussian measure density doesn't demand the use of analytic expressions for Fourier transforms $\Phi_{\lambda}(\vec{k})$. But for integration with the non-Gaussian measure density such an expression is necessary. So, we shall use the following representations [2]:

$$
\begin{aligned}
& \Phi_{1}(\vec{k})=J_{11}(\vec{k})+J_{12}(\vec{k})=\varphi_{0}-\lambda \cos ^{2} \vartheta-A|\vec{k}|^{2}, \\
& \Phi_{5}(\vec{k})=J_{11}(\vec{k})-J_{12}(\vec{k}),
\end{aligned}
$$

where $\vartheta$ is a polar angle in the ellipsoid of revolution coordinate system, $\varphi_{0}, \lambda, A$ are some constants.

After expanding the exponent containing $\mathcal{M}_{5555}\left(\vec{k}_{1}, \nu_{1}, \ldots, \vec{k}_{4}, \nu_{4}\right)$ and integrating every addendum with Gaussian measure density $(8)$ over $\rho_{5}(\vec{k}, \nu)$, for a non-active transition branch of the system in the ferroelectric phase, we obtain:

$$
\begin{gathered}
Z_{5}^{G}=\prod_{\vec{k}, \nu}\left\{\sqrt{1-\beta \Phi_{5}(0) \mathcal{M}_{55}(0, \nu)}\left(1-\beta \Phi_{5}(\vec{k}) \mathcal{M}_{55}(\vec{k}, \nu)\right)\right\}^{-1} \\
\times \exp \left\{\frac{1}{8} \sum_{k, \nu, k^{\prime}, \nu^{\prime}} \mathcal{M}_{5555}\left(\vec{k}, \nu,-\vec{k},-\nu, \vec{k}^{\prime}, \nu^{\prime},-\vec{k}^{\prime},-\nu^{\prime}\right) g_{5}(\vec{k}, \nu) g_{5}\left(\vec{k}^{\prime}, \nu^{\prime}\right)\right\},
\end{gathered}
$$

where

$$
g_{5}(\vec{k}, \nu)=\beta \Phi_{5}(\vec{k})\left(1-\beta \Phi_{5}(\vec{k}) \mathcal{M}_{55}(\vec{k}, \nu)\right)^{-1}
$$

is a Fourier transform of the screened potential of the $\lambda=5$ branch.

Integration of $(2)$ over $\rho_{1}(\vec{k}, \nu)$ at $\nu \neq 0$, in principle, may be performed using the Gaussian measure density (expression (9) at $\mathcal{M}_{1111}\left(\vec{k}_{1}, \nu_{1}, \ldots, \vec{k}_{4}, \nu_{4}\right)=0$ ). It brings into the total $Z$ an additional factor $Z_{1}^{G}$ similar to (11), in which $5 \rightarrow 1$ and $\nu \neq 0$. So, it remains to integrate (2), using quartic measure density, only over the collective variables

$$
\rho_{k} \equiv \rho_{1}(\vec{k}, \nu=0) \text {. }
$$

For factorization of the integrand in $(2)$ with respect to variables $\rho_{k}$ let's introduce the node variables:

$$
\begin{aligned}
& \rho_{l}=\frac{1}{\sqrt{N}} \sum_{k} \rho_{k} \mathrm{e}^{\mathrm{i} \vec{k} \vec{l}}, \\
& \omega_{l}=\frac{1}{\sqrt{N}} \sum_{k} \omega_{k} \mathrm{e}^{-\mathrm{i} \vec{k} \vec{l}}, \quad\left(\mathrm{~d} \omega_{k}\right)^{N}=\mathrm{d} \omega_{0} \prod_{k>0} \mathrm{~d} \omega_{k}^{s} \mathrm{~d} \omega_{k}^{c}=\sqrt{2} \prod_{l}^{N-1} \mathrm{~d} \omega_{l} .
\end{aligned}
$$

The (2) expression takes the following form:

$$
Z=Z_{0}^{N} Z_{5}^{G} Z_{1}^{G} Z_{1}
$$


where

$$
\begin{aligned}
Z_{1}= & \sqrt{2}^{N-1} Q^{N} \int\left(\mathrm{d} \rho_{k}\right)^{N} \exp \left\{\frac{\beta}{2} \Phi_{1}(\vec{k}) \rho_{k} \rho_{-k}-2 \sqrt{N} \beta h \rho_{k} \delta(\vec{k})\right\} \\
& \times \sqrt{2}^{N-1} Q \prod_{l}\left\{-\frac{1}{2} a_{2} \rho_{l}^{2}-\frac{1}{4 !} a_{4} \rho_{l}^{4}\right\} \\
Q= & 2 \int_{0}^{\infty} f^{q}(\omega) \mathrm{d} \omega \\
a_{2}= & (2 \pi)^{2} Q^{-1} \int_{-\infty}^{\infty} \omega^{2} f^{q}(\omega) \mathrm{d} \omega \\
a_{4}= & -(2 \pi)^{4} Q^{-1} \int_{-\infty}^{\infty} \omega^{4} f^{q}(\omega) \mathrm{d} \omega+3 a_{2}^{2} .
\end{aligned}
$$

The method of integration of the partition function functional critical part (16) was developed in [3,7]. The main peculiarity of this method is a twofold character of integration process with the use of the Gaussian and the non-Gaussian measure densities. Due to the unisotropic dipole-dipole potential $\Phi_{1}(\vec{k})(10)$ one may find in the first Brillouin zone such a set of wave vectors $\vec{k}$, for which variables $\rho_{k}$ are distributed with the Gaussian measure density. Corresponding coefficient in (16)

$$
d_{2}(\vec{k})=a_{2}-\beta \Phi_{1}(\vec{k})
$$

for those $\vec{k}$ is positive. The rest of variables $\rho_{\vec{k}}$ should be considered in accordance with the non-Gaussian form of their distribution. We had proposed to perform this two-stage integration in every layer of Brillouin zone when layer by layer integration method is used $[1,7]$.

As a result, after $(n-1)$ stage of integration the $Z_{1}$ takes the following form:

$$
\begin{aligned}
Z_{1}= & \sqrt{2}^{N-1} Q^{N} \prod_{m=1}^{n-1}\left\{\sqrt{2}^{-N_{m}} C_{m}\left[Q\left(\frac{d_{2}^{(m)}\left(\frac{B_{m}}{s}, B_{m}\right)}{(2 \pi)^{2}}, \frac{a_{4}^{(m)}}{(2 \pi)^{4}}\right)\right]^{N_{m}-N_{m}^{G}}\right. \\
& \left.\times\left[Q\left(P_{2}^{(m)}, P_{4}^{(m)}\right)\right]^{N_{m+1}}\right\} \int\left(\mathrm{d} \rho_{k}\right)^{N_{n}} \exp \left\{-\frac{1}{2} \sum_{k \in B_{n}} d_{2}^{(n)}(\vec{k}) \rho_{k} \rho_{-k}\right. \\
& \left.-\frac{1}{4 ! N_{n}} \sum_{k_{1}, \ldots, k_{4} \in B_{n}} a_{4}^{(n)} \rho_{k_{1}} \ldots \rho_{k_{4}} \delta\left(\vec{k}_{1}+\ldots+\vec{k}_{4}\right)-2 \sqrt{N} \beta h \rho_{k} \delta(\vec{k})\right\} .
\end{aligned}
$$

Here $Q\left(\frac{d_{2}^{(m)}\left(\frac{B_{m}}{s}, B_{m}\right)}{(2 \pi)^{2}}, \frac{a_{4}^{(m)}}{(2 \pi)^{4}}\right), Q\left(P_{2}^{(m)}, P_{4}^{(m)}\right)$ are certain combinations of modified Bessel functions of $z_{m}$ argument [3].

$$
z_{m}=\frac{3\left[d_{2}^{(m)}\left(\frac{B_{m}}{s}, B_{m}\right)\right]^{2}}{a_{4}^{(m)}}, \quad d_{2}^{(m)}\left(\frac{B_{m}}{s}, B_{m}\right)=\left\langle d_{2}^{(m)}(\vec{k})\right\rangle_{\frac{B_{m}}{s}, B_{m}}
$$




$$
\begin{aligned}
C_{m} & =\prod_{k \in B_{m}^{G}}\left\{\frac{\pi}{d_{2}^{(m)}(\vec{k})}\right\} \exp \left\{-\frac{a_{4}^{(m)}}{8 N} \sum_{k_{1}, k_{2} \in B_{m}^{G}} \frac{1+\delta_{k_{1} k_{2}}}{d_{2}^{(m)}\left(\vec{k}_{1}\right) d_{2}^{(m)}\left(\vec{k}_{2}\right)}\right\}, \\
P_{2}^{(m)} & =\sqrt{\frac{12}{a_{4}^{(m)}}} \mathcal{K}\left(z_{m}\right), \quad P_{4}^{(m)}=\frac{N_{m+1}}{N_{m}-N_{m}^{G}} \frac{6}{a_{4}^{(m)}} L\left(z_{m}\right), \\
\mathcal{K}\left(z_{m}\right) & =\sqrt{z_{m}}\left(\frac{K_{3 / 4}\left(z_{m}\right)}{K_{1 / 4}\left(z_{m}\right)}-1\right), \quad L\left(z_{m}\right)=6 \mathcal{K}^{2}\left(z_{m}\right)+4 \sqrt{z_{m}} \mathcal{K}\left(z_{m}\right)-1, \\
N_{m} & =\frac{N}{s^{3(m-1)}} \sqrt{\frac{1}{\beta \lambda}\left(\beta \varphi_{0}-a_{2}^{(m-1)}\right)}, \\
N_{m}^{G} & =\frac{N}{s^{3(m-1)}}\left[\sqrt{\frac{1}{\beta \lambda}\left(\beta \varphi_{0}-a_{2}^{(m-1)}\right)}-\sqrt{\frac{1}{\beta \lambda}\left(\beta \varphi_{0}-a_{2}^{(m)}\right)}\right],
\end{aligned}
$$

$s=\left|B_{n}\right| /\left|B_{n+1}\right|,\left|B_{n}\right|$ is an effective "Brillouin zone" radius at the $n$-th step of layer by layer integration.

Quantities $d_{2}^{(m)}\left(\frac{B_{m}}{s}, B_{m}\right)$ and $a_{4}^{(m)}$ satisfy certain recursion relations, which completely characterizes the ferroelectric cluster system behaviour in the phase transition point neighbourhood. Linearization of those relations near fixed point allows us to obtain the expressions for $d_{2}^{(m)}\left(\frac{B_{m}}{s}, B_{m}\right), a_{4}^{(m)}$ in the evident form:

$$
\begin{aligned}
d_{2}^{(m)}\left(\frac{B_{m}}{s}, B_{m}\right) & =\frac{c_{1} E_{1}^{m-1}-c_{2} R E_{2}^{m-1}+q}{s^{2(m-1)}}, \\
a_{4}^{(m)} & =\frac{c_{1} R^{\prime} E_{1}^{m-1}+c_{2} E_{2}^{m-1}}{s^{4(m-1)}},
\end{aligned}
$$

where $c_{1}=\tilde{c}_{1} \tau \ln ^{-1 / 3}|\tau| ; E_{1}, E_{2}$ are the eigenvalues of the linear transformation matrix of the above mentioned recursion relations $\left(E_{1}=s^{2}, E_{2}=1\right) ; q=\frac{3}{5} \frac{1-s^{-5}}{1-s^{-3}} \beta A \pi^{2}$; $\tilde{c}_{1}, c_{2}, R, R^{\prime}$ are constants, which are determined by the linear transformation matrix; $\tau=T-T_{\mathrm{c}} / T_{\mathrm{c}}[7]$.

At $T<T_{\mathrm{c}}$ integration in (19) is performed up to $n=\mu_{\tau}$, which may be determined from the relation

$$
d_{2}^{\left(\mu_{\tau}\right)}\left(|\vec{k}|=\left|B_{\mu_{\tau}}\right|\right)=0
$$

and

$$
\mu_{\tau}=1+\ln \frac{c_{2} R-q}{c_{1}} / \ln E_{1} .
$$

Assuming that layer by layer integration in (19) has been performed over all $\rho_{k}$ with $|\vec{k}| \geqslant\left|B_{\mu_{\tau}}\right|$, let's pass to the last stage of integration (over $\rho_{k}$ with $|\vec{k}|<\left|B_{\mu_{\tau}}\right|$ ). It may be proved that in this region of collective variables $\rho_{k}$ are distributed according to Gaussian law, but with non-analytic coefficient, so-called, inverse Gaussian regime. In order to show this fact, we should take into account the shift of the fluctuation center of the $\rho_{k}$ in the ordered phase:

$$
\rho_{k}=\rho_{k^{\prime}}+\sqrt{N}\langle\sigma\rangle \delta(\vec{k}) .
$$


As a result, the quartic form (19) changes and becomes as follows:

$$
\begin{aligned}
E_{\mu_{\tau}}(\rho)= & -\frac{1}{2} \sum_{k} d_{2}^{\left(\mu_{\tau}\right)}(\vec{k}) \rho_{\vec{k}} \rho_{-\vec{k}}-\frac{a_{4}^{\left(\mu_{\tau}\right)}}{4 ! N_{\mu_{\tau}}} \sum_{k_{i}}^{\prime} \rho_{k_{1}} \rho_{k_{2}} \rho_{k_{3}} \rho_{k_{4}} \\
& -\frac{a_{4}^{\left(\mu_{\tau}\right)}}{3 ! N_{\mu_{\tau}}}\left(\rho_{0}+\sqrt{N}\langle\sigma\rangle\right) \sum_{k_{i}}^{\prime} \rho_{k_{1}} \rho_{k_{2}} \rho_{k_{3}} \\
& -\frac{a_{4}^{\left(\mu_{\tau}\right)}}{4 ! N_{\mu_{\tau}}}\left(\rho_{0}^{2}+2 \sqrt{N} \rho_{0}\langle\sigma\rangle+N\langle\sigma\rangle^{2}\right) \sum_{k_{i}}^{\prime} \rho_{k} \rho_{-k} \\
& -\frac{a_{4}^{\left(\mu_{\tau}\right)}}{4 ! N_{\mu_{\tau}}} \rho_{0}^{4}-\frac{a_{4}^{\left(\mu_{\tau}\right)}}{3 ! N_{\mu_{\tau}}} \sqrt{N}\langle\sigma\rangle \rho_{0}^{3}-\frac{a_{4}^{\left(\mu_{\tau}\right)}}{4 ! N_{\mu_{\tau}}} \sqrt{N}\langle\sigma\rangle^{2} \rho_{0}^{2} \\
& -\sqrt{N}\left(2 \beta h+d_{2}^{\left(\mu_{\tau}\right)}(0)\langle\sigma\rangle+\frac{a_{4}^{\left(\mu_{\tau}\right)}}{3 ! N_{\mu_{\tau}}} N\langle\sigma\rangle^{3}\right) \rho_{0} \\
& -N\left(2 \beta h\langle\sigma\rangle+\frac{d_{2}^{\left(\mu_{\tau}\right)}(0)}{2}\langle\sigma\rangle^{2}+\frac{a_{4}^{\left(\mu_{\tau}\right)}}{4 ! N_{\mu_{\tau}}} N\langle\sigma\rangle^{4}\right) .
\end{aligned}
$$

Here $\sum_{k}^{\prime}$ means summation over all $|\vec{k}| \leqslant\left|B_{\mu_{\tau}}\right|$ with the exception of $k=0$.

The last term in (25) is a macroscopic part of the "Hamiltonian" $E_{\mu_{\tau}}(\rho)$. The shift parameter $\langle\sigma\rangle$ is to be determined from the condition of minimum of this part. With the accuracy up to the first order of $h$ we obtained:

$$
\langle\sigma\rangle=\left(\frac{3 !\left|d_{2}^{\left(\mu_{\tau}\right)}(0)\right| N_{\mu_{\tau}}}{a_{4}^{\left(\mu_{\tau}\right)} N}\right)^{1 / 2}-\frac{\beta h}{\left|d_{2}^{\left(\mu_{\tau}\right)}(0)\right|} .
$$

Inserting $\langle\sigma\rangle$ into (25), we obtain the following expression for $E_{\mu_{\tau}}(\rho)$ :

$$
\begin{aligned}
E_{\mu_{\tau}}(\rho)= & -\frac{1}{2} \sum_{k}^{\prime} \bar{d}_{2}(\vec{k}) \rho_{\vec{k}} \rho_{-\vec{k}}-\frac{a_{4}^{\left(\mu_{\tau}\right)}}{4 ! N_{\mu_{\tau}}} \sum_{k}^{\prime} \rho_{k_{1}} \rho_{k_{2}} \rho_{k_{3}} \rho_{k_{4}} \\
& -\left[\frac{a_{4}^{\left(\mu_{\tau}\right)}}{3 ! N_{\mu_{\tau}}} \rho_{0}+\left(\frac{\bar{d}_{2}(0) a_{4}^{\left(\mu_{\tau}\right)}}{2 \cdot 3 ! N_{\mu_{\tau}}}\right)^{1 / 2}\right] \sum_{k}^{\prime} \rho_{k_{1}} \rho_{k_{2}} \rho_{k_{3}} \\
& -\frac{1}{2}\left[\frac{a_{4}^{\left(\mu_{\tau}\right)}}{2 N_{\mu_{\tau}}} \rho_{0}^{2}+\left(\frac{3 \bar{d}_{2}(0) a_{4}^{\left(\mu_{\tau}\right)}}{N_{\mu_{\tau}}}\right)^{1 / 2} \rho_{0}\right] \sum_{k}^{\prime} \rho_{k} \rho_{-k} \\
& -\frac{a_{4}^{\left(\mu_{\tau}\right)}}{4 ! N_{\mu_{\tau}}} \rho_{0}^{4}-\left(\frac{\bar{d}_{2}(0) a_{4}^{\left(\mu_{\tau}\right)}}{2 \cdot 3 ! N_{\mu_{\tau}}}\right)^{1 / 2} \rho_{0}^{3}-\frac{\bar{d}_{2}(0)}{2} \rho_{0}^{2}-2 \sqrt{N} \beta h \rho_{0}+M_{\text {mac }} .
\end{aligned}
$$

Here

$$
M_{\mathrm{mac}}=\frac{3}{8 a_{4}^{\left(\mu_{\tau}\right)}}\left(\bar{d}_{2}(0)\right)^{2} N_{\mu_{\tau}}-\left(\frac{2 \cdot 3 ! \bar{d}_{2}(0) N_{\mu_{\tau}} N}{a_{4}^{\left(\mu_{\tau}\right)}}\right)^{1 / 2} \beta h
$$




$$
\bar{d}_{2}(\vec{k})=3\left|d_{2}^{\left(\mu_{\tau}\right)}(0)\right|+d_{2}^{\left(\mu_{\tau}\right)}(\vec{k})=2\left|d_{2}^{\left(\mu_{\tau}\right)}(0)\right|+q k^{2} .
$$

So, the functional $\exp \left\{E_{\mu_{\tau}(\rho)}\right\}$ will now be integrated with the well-defined Gaussian form:

$$
\exp \left\{-\frac{1}{2} \sum_{k}^{\prime} \bar{d}_{2}(\vec{k}) \rho_{k} \rho_{-k}\right\}
$$

and $\bar{d}_{2}(0)$, due to the previous integration, is a non-analytic function of $\tau$ (see (21)). With the accuracy up to the first order of the inverse Bessel function argument $z_{\mu}^{-1}$ after integration of the $\exp \left\{E_{\mu_{\tau}(\rho)}\right\}$ over all variables, with the exception $\rho_{0}$, we obtained:

$$
\begin{aligned}
& Z_{\mu}=\exp \left\{-\beta\left(F_{\mathrm{mac}}+F_{\mathrm{RPA}}+\Delta F_{\mu_{\tau}}+F_{g}\right)\right\} \\
& \times \int \exp \left\{-N\left[D \rho^{4}-B \rho^{2}-A \rho\right]\right\} \mathrm{d} \rho .
\end{aligned}
$$

Here

$$
\begin{aligned}
& -\beta F_{\mathrm{mac}}=\frac{3}{8} \frac{\bar{d}_{2}^{2}(0) N_{\mu_{\tau}}}{a_{4}^{\left(\mu_{\tau}\right)}}-\left(\frac{2 \cdot 3 ! \bar{d}_{2}(0) N_{\mu_{\tau}} N}{a_{4}^{\left(\mu_{\tau}\right)}}\right)^{1 / 2} \beta h, \\
& -\beta F_{\mathrm{RPA}}=-\frac{1}{2} \sum_{k}^{\prime} \ln \frac{\bar{d}_{2}(\vec{k})}{\pi}, \\
& -\beta \Delta F_{\mu_{\tau}}=\left\langle\rho_{0}\right\rangle+\frac{1}{2}\left(\left\langle\rho_{0}^{2}\right\rangle-\left\langle\rho_{0}\right\rangle^{2}\right), \\
& \left\langle\rho_{0}\right\rangle=\frac{1}{8} a_{4}^{\left(\mu_{\tau}\right)}\left(\frac{1}{N_{\mu_{\tau}}} \sum_{k}^{\prime} \frac{1}{\bar{d}_{2}(\vec{k})}\right)^{2}, \\
& \left\langle\rho_{0}^{2}\right\rangle=\frac{1}{2} \bar{d}_{2}(0) a_{4}^{\left(\mu_{\tau}\right)} \frac{1}{N_{\mu_{\tau}}}\left(\sum_{k}^{\prime} \frac{\mathrm{e}^{\mathrm{i} \vec{k} \vec{r}}}{\bar{d}_{2}(\vec{k})}\right)^{3}, \\
& -\beta F_{g}=\sqrt{\frac{2 \cdot 3 ! \bar{d}_{2}(0) N_{\mu_{\tau}} N}{a_{4}^{\left(\mu_{\tau}\right)}}} \beta h ; \\
& A=2 \beta h, \quad B=\frac{1}{4}\left(\bar{d}_{2}(0)-\frac{a_{4}^{\left(\mu_{\tau}\right)}}{N_{\mu_{\tau}}} \sum_{k}^{\prime} \frac{1}{\bar{d}_{2}(\vec{k})}\right), \quad D=\frac{a_{4}^{\left(\mu_{\tau}\right)}}{4 !} \frac{N}{N_{\mu_{\tau}}} ; \\
& \rho=\sqrt{N} \rho_{0} .
\end{aligned}
$$

We would like to note that the way of integration over $\rho_{k}$ with $\vec{k}$ smaller $B_{\mu_{\tau}}$, presented here, for the other types of interacting many-particle systems, was used in $[6,8]$.

The minimization procedure of integrand in (31) gives an equation for determining the order parameter (spontaneous polarization):

$$
4 D \rho^{3}-2 B \rho-A=0 .
$$


Before solving this equation we should determine the temperature dependence of $A, B, D$ coefficients. Using expressions (32) and (21) one may find:

$$
\begin{aligned}
& A=2 \beta h, \\
& B=\frac{\tilde{c}_{1}}{2}\left(1-\frac{c_{2} R}{c_{2} R-q}+\frac{3}{4} \frac{c_{2}\left(R R^{\prime}+1\right)-q R^{\prime}}{\left(c_{2} R-q\right) q} \frac{t-\arctan t}{t^{3}}\right) \tau \ln ^{-1 / 3}|\tau|, \\
& D=\frac{1}{4 !}\left(c_{2}\left(R R^{\prime}+1\right)-q R^{\prime}\right) \ln ^{-2 / 3}|\tau|, \quad t=\frac{\pi}{b} \sqrt{\frac{\beta \varphi_{0}}{2 c_{2} R}}
\end{aligned}
$$

$b$ is a cubic lattice constant. It should be noted that to calculate the quantity $\frac{N}{N_{\mu}}=s^{4\left(\mu_{\tau}-1\right)}$ in $D$ (see formulae (2), (21), (23) and (32)), the $d_{2}^{\left(\mu_{\tau}\right)}(0)$ coefficient non-renormalized by integration with the Gaussian measure density was used. So, $s^{4\left(\mu_{\tau}-1\right)}=\left(\frac{c_{2} R-q}{\tilde{c}_{1}}\right)^{2}|\tau|^{-2}$ in this case.

Introducing new coefficients:

$$
\begin{aligned}
& v=\frac{9 \tilde{c}_{1}}{2 q\left(c_{2} R-q\right)}\left(\frac{t-\arctan t}{t^{3}}-\frac{4 q^{2}}{3\left(c_{2}\left(R R^{\prime}+1\right)-q R^{\prime}\right)}\right), \\
& w=\frac{12}{c_{2}\left(R R^{\prime}+1\right)-q R^{\prime}},
\end{aligned}
$$

we have obtained the final expression for a spontaneous polarization equation of the ferroelectric cluster system:

$$
\rho^{3}-v \tau \ln ^{1 / 3}|\tau| \rho-w \ln ^{2 / 3}|\tau| \beta h=0 .
$$

According to the Cardan formula [9], the equation (36) has the following roots:

$$
\rho_{1}=A+B, \quad \rho_{2,3}=-\frac{A+B}{2} \pm \mathrm{i} \sqrt{3} \frac{A-B}{2},
$$

where

$$
\begin{aligned}
& A=\sqrt[3]{3 w \ln ^{2 / 3}|\tau| \beta h+\sqrt{R}}, \quad B=\sqrt[3]{3 w \ln ^{2 / 3}|\tau| \beta h-\sqrt{R}} \\
& R=-\left(\frac{v}{3}\right)^{3} \tau^{3} \ln |\tau|+w^{2} \ln ^{4 / 3}|\tau| \frac{\beta^{2} h^{2}}{2}
\end{aligned}
$$

At $T<T_{\mathrm{c}}$ :

a) the external field is missing $(h=0, R<0)$. Equation (37) has three different roots:

$$
\rho_{1}=v^{1 / 2}|\tau|^{1 / 2} \ln ^{1 / 6}|\tau|, \quad \rho_{2}=-v^{1 / 2}|\tau|^{1 / 2} \ln ^{1 / 6}|\tau|, \quad \rho_{3}=0 .
$$

b) the external field is small $(h \neq 0, R<0)$. Equation (37) has three roots non-equal to zero.

c) the external field is large $(h \neq 0, R>0)$. Equation (37) has one real and two complex-conjugated roots. Real root is approximately equal to

$$
\rho=w^{1 / 3} \ln ^{2 / 9}|\tau|(\beta h)^{1 / 3} .
$$


At $T>T_{\mathrm{c}}$ :

a) the external field is missing $(h=0, R>0)$. Equation (37) has one real:

$$
\rho=0
$$

and two complex-conjugated roots.

b) the external field non-equal to zero $(h \neq 0, R>0)$. Equation $(37)$ has got a real root and two complex-conjugated roots as in (40).

The precise analysis of the spontaneous polarization behaviour near the phase transition point will be done with the help of derivations $\partial \rho / \partial \tau$ and $\partial \rho / \partial \beta h$. Differentiating equation (36) with respect to $\tau$ and $\beta h$, we obtain:

$$
\begin{aligned}
& \frac{\partial \rho}{\partial \tau}=\frac{v \rho\left(1+\frac{1}{3} \ln ^{-1}|\tau|\right)+\frac{2 w}{3 \tau} \ln ^{-2 / 3}|\tau| \beta h}{3 \rho^{2} \ln ^{-1 / 3}|\tau|-v \tau}, \\
& \frac{\partial \rho}{\partial \beta h}=\frac{w \ln ^{1 / 3 \mid} \tau \mid}{3 \rho^{2} \ln ^{-1 / 3}|\tau|-v \tau} .
\end{aligned}
$$

It has been seen that logarithmic corrections help to attain a sharper behaviour of the $\rho(\tau)$ near $\tau=0$, both at $h=0$ and $h \neq 0$. But at $h=0$ the $\rho(\tau)$ increases more rapidly as compared with the case $h \neq 0$. This is due to the fact that $\rho(0)=0$ at $h=0$ and $\rho(0) \neq 0$ at $h \neq 0$.

The peculiarities of the $\rho(\tau)$ behaviour near $T_{\mathrm{c}}$ are connected with the quantity of the dielectric susceptibility $\chi$ in this region. Using the expressions (39), (42) the formula for $\chi$ is easy to obtain:

$$
\chi=\beta\left(\frac{\partial \rho}{\partial \beta h}\right)_{h=0}= \begin{cases}\chi_{+}=-\frac{\beta w \ln ^{1 / 3}|\tau|}{v \tau}, & \tau>0 \\ \chi_{-}=\frac{\beta w \ln ^{1 / 3}|\tau|}{2 v \tau}, & \tau<0\end{cases}
$$

In (43) we have kept the terms containing only the leading power of $\tau$.

One may verify that qualitatively (43) and the corresponding expression, obtained phenomenologically based on the Gaussian summation in [10], are similar. The "law of double" $\left(\chi_{+}=2 \chi_{-}\right)$is also fulfilled.

\section{References}

1. Yukhnovskii I.R. Phase Transition of the Second Order. Collective Variables Method. Singapore, World Scientific, 1987.

2. Yukhnovskii I.R., Korynevskii N.A. // Phys. Stat. Sol. (b), 1989, vol. 153, p. 583.

3. Yukhnovskii I.R., Korynevskii N.A. // Phys. Stat. Sol. (b), 1989, vol. 154, p. 519.

4. Hubbard J. // Proc. Roy. Soc., 1965, vol. A285, p. 542.

5. Didukh L.D., Stasyuk I.V. // Phys. Met. and Metalloved., 1968, vol. 26, p. 582 (in Russian).

6. Korynevskii N.A. Order parameter of a ferromagnetic phase transition in the twoparticle magnetic cluster system. Preprint of the Institute for Condensed Matter Physics, ICMP-99-01U, Lviv, 1999, 24 p. (in Ukrainian). 
7. Korynevskii N.A. // Ferroelectrics, 1997, vol. 192, p. 45.

8. Yukhnovskii I.R. // Nuovo Cimento, 1989, vol. 12, p. 112.

9. Korn G.A., Korn T.M. Mathematical handbook for scientists and engineers. N.J.-T.L., eMGRAW-Hill Book Com. inc., 1961.

10. Larkin A.I., Khmelnitskii D.E. // JETP, 1969, vol. 56, p. 2087 (in Russian).

\title{
Спонтанна поляризація в кластерній сегнетоелектричній системі поблизу точки фазового переходу
}

\author{
М.А.Кориневський \\ Інститут фізики конденсованих систем НАН України, \\ 79011 Львів, вул. Свєнціцького, 1 \\ Отримано 22 квітня 1999 р.
}

В наближенні четвірного базисного розподілу фаз флуктуацій дипольного моменту сегнетоелектричної двочастинкової кластерної системи виконано двоетапне пошарове інтегрування функціонала статистичної суми. Форма шарів інтегрування визначається особливостями флуктуаційних процесів (гаусового і негаусового типу) окремих підмножин колективних змінних. Мінімізація недоінтегрованого за однією змінною (яка характеризується нульовими значеннями квазіімпульса і мацубарівської частоти) функціонала статистичної суми визначає рівняння для параметра порядку (спонтанної поляризації) системи. Знайдено розв'язки цього рівняння при різних значеннях температури (в околі $T_{\mathrm{c}}$ ), параметрів системи і зовнішнього поля.

Ключові слова: функціонал, кластерні сегнетоелектрики, фазовий перехід, поляризація

PACS: $75.40 . C x$ 
